\title{
La perspectiva feminista de género en la migración laboral internacional
}

\author{
The feminist perspective on gender \\ in international labor migration
}

Genoveva Roldán Dávila*

ISSN IMPRESO 1870-7599 | ISSN RED CÓMPUTO 2448-7783 | 25-44

RECIBIDO: 08/08/2018 | ACEPTADO: 13/08/2018

Resumen. Las migraciones internacionales están articuladas por diversas estructuras sociales discriminatorias, signadas por clase social, etnia, edad, nacionalidad. Al respecto, en este artículo se visualiza la presencia de las mujeres marcada además por las condiciones de desigualdad de género. El objetivo de contribuir en el debate se orienta a cuestionar la percepción social androcéntrica y sexista que, empírica o teóricamente, invisibiliza su presencia o la representa en condiciones de subsidiariedad y adhesión a la protagonizada por los hombres. La feminización de las migraciones laborales internacionales es parte esencial de las singularidades que exhibieron las dinámicas migratorias, a partir de la reestructuración capitalista que tuvo auge en las dos últimas décadas del siglo XX y que en el presente siglo, pese a la crisis de 2008, el endurecimiento de las políticas antimigratorias y de la xenofobia populista, no se han materializado en su disminución. Esta situación puede observarse en particular en el gran sistema migratorio constituido por Estados Unidos y mujeres y hombres latinoamericanos.

Palabras clave: migración internacional, mujeres, feminización, Estados Unidos, latinas y latinos.

Abstract. International migrations are tied to various discriminatory social structures, characterized by social class, ethnicity, age, nationality; this article addresses women who are also affected by conditions of gender inequality. The aim in contributing to the debate is to question the androcentric and sexist social perception that, empirically or theoretically, masks their presence or places women in a subsidiary position and under the domination of men. The feminization of international labor migration is an essential characteristic of the singularities that are found in migration dynamics, through the capitalist restructuring that developed in the last two decades of the 20th Century and that in the contemporary period stems from the 2008 crisis, the hardening of anti-migratory policies and populist xenophobia, which exhibit no alleviation. This is the context of today's broad migratory system that encompasses the United States and Latin American men and women.

Keywords: international migration, women, feminization, United States, Latin Americans. 


\section{Introducción}

En el último lustro el análisis y el debate de los procesos migratorios ocupan los primeros lugares de las agendas institucionales y políticas de Estados de origen, tránsito y destino, organismos internacionales, organizaciones sociales e instituciones académicas. En no pocas ocasiones aluden a ellos las principales portadas de los medios de comunicación virtuales e impresos, incluso ganan premios por fotografías que evidencian, de manera descarnada, las condiciones límites que enfrentan en la migración mujeres, hombres, niños y niñas, ya sea como resultado de las propias dinámicas migratorias o de aquellas que derivan de conflictos bélicos, crisis económicas y procesos electorales. Cada vez más, amplios sectores sociales, partidos políticos y líderes conservadores de gran trascendencia mundial, convierten estos procesos en "problemas públicos», equiparados con pandemias y amenazas que rompen la tan anhelada "armonía» social, la cual se encuentra más alejada de las condiciones resultantes del modelo de desarrollo vigente.

En ese marco de convulsas condiciones, el objetivo del artículo es visibilizar la presencia de las mujeres en migración, marcada por las estructuras discriminatorias en las que están inscritas las migraciones en general y por las condiciones de desigualdad de género. En las dos últimas décadas del siglo XX y las otras casi dos décadas del presente siglo ha tenido lugar un intenso debate, todavía en curso, acerca de cuál es el contenido de un concepto identificado como la feminización de las migraciones. La orientación central del planteamiento cuestiona la percepción social androcéntrica y sexista que, en la teoría y en el conocimiento empírico, o bien han invisibilizado su presencia o la han representado en condiciones de subsidiariedad y adhesión a la protagonizada por los hombres.

De tal forma, no resulta ocioso cuestionarse en torno de cuál es el nivel de comprensión que socialmente existe sobre los procesos migratorios de mujeres y hombres, en particular los laborales (63.5 por ciento del total de migrantes) de importancia significativa por el alcance económico, social y cultural que tienen en tanto inmigrantes, así como en sus familias, comunidades y países. Referente al total de migrantes, se calculan en 258 millones, de ellos 164 millones son trabajadores (ILO, 2018). Del total de los trabajadores migrantes, 68 millones son mujeres y representan casi 42 por ciento. Los datos previos subestiman la realidad, pues aun cuando el empleo no se encuentre en la explicación fundamental de otras movilidades, aparecerá en algún momento del proceso migratorio (OIT, 2017), es el caso de la reunificación familiar, el refugio y el asilo. 
Además de los discursos xenófobos ya mencionados, a los migrantes laborales también se les señala por «quitar trabajo a los nativos» o por solicitar refugio o asilo a través de falsedades, cuando en realidad sólo son «inmigrantes económicos». Javier de Lucas (1996) insiste en que tales señalamientos identifican a la migración laboral como menos «digna» o radicalmente diferente de quien es perseguido político; no obstante, en la realidad las diversas modalidades migratorias se entretejen por contextos difícilmente divisibles. Se ha corroborado que los inmigrantes no ocupan ni compiten por los empleos destinados en los mercados laborales a los nativos y que la evolución demográfica de los países industrializados se expresa en el envejecimiento de su población, de ahí que los trabajadores inmigrantes se concentren en esos países. De los 164 millones de trabajadores migrantes, 111.2 millones (68 por ciento) se emplea en esos países (ILO, 2018); su presencia es significativa porque asciende a 18.5 por ciento del total de la fuerza laboral.

Este trabajo forma parte del pensamiento que enfoca la investigación sobre la feminización de las migraciones laborales internacionales como un proceso que es pieza esencial de las singularidades que exhibieron las dinámicas migratorias, a partir de la reestructuración capitalista que cobró auge en las dos últimas décadas del siglo XX. Se sostiene la hipótesis de que el detonante de dicha feminización son las grandes tendencias presentadas por los mercados laborales de los países de destino: terciarizados, flexibles y, de modo relevante, generizados, que requieren de tal fuerza de trabajo; proceso que se sustenta en la existencia de una complementariedad subordinada del mercado laboral mexicano con el estadounidense. Dinámica que también se sostiene en los nuevos entornos económicos e ideológicos que, sobre la actuación y contextos de inclusión de las mujeres en la reproducción productiva-social, se han observado en ese periodo. Las especificidades de la incorporación de las mujeres latinas como trabajadoras en la economía estadounidense explica por qué, aun con la crisis de 2008, el endurecimiento de las políticas antimigratorias y el incremento de la xenofobia populista frente a la inmigración no ha disminuido su demanda.

En síntesis, casi telegráficamente y en calidad de prontuario, en un primer inciso se abordarán los grandes muros ideológicos que han limitado el conocimiento científico de las migraciones laborales de mujeres y hombres. En segundo término se planteará un conjunto de reflexiones que dilucidan la comprensión sobre la feminización de las migraciones. El tercer inciso tiene como propósito presentar las peculiaridades de la incorporación de las mujeres latinas trabajadoras 
en el mercado laboral estadounidense y explicar su permanente crecimiento. Por último, se expondrán las conclusiones más destacadas.

\section{Los muros ideológicos. Contexto y causas de las migraciones laborales internacionales}

Las políticas migratorias se siguen respaldando en el mainstream, en la ortodoxia que ha sido dominante en las teorías migratorias, de tal manera que el marco interpretativo gira alrededor del análisis de las condiciones que desde los países de origen empujan a la migración y en todos aquellos elementos que influyen en la decisión individual o de estrategias familiares, que evidencian la libre decisión, la agencia, la capacidad individual de resistir ante las condiciones estructurales, su emprendimiento, el estímulo que obtienen a su capital humano y, como colofón, los diversos beneficios que sus comunidades, regiones y países obtienen.

Aceptar el discurso expandido mundialmente del vecino del norte, en cuanto a que las insuficiencias y debilidades de los mercados laborales, la marginación y la violencia agudizada en los países de origen son los elementos que catalizan los flujos migratorios, se traduce en una aceptación implícita del discurso conservador que estuvo claramente representado en 2004 por Samuel P. Huntington, miembro del selecto círculo académico de Harvard. Él insiste en que el enemigo de Estados Unidos se encuentra en su interior, como resultado de una «invasión silenciosa» de los inmigrantes latinoamericanos y, en concreto, de la procedente de México.

En teoría, este discurso se distancia de la anterior perspectiva: la propuesta teórica del push \& pull; sin embargo, aparte de que está influido por un mecanicismo y un enfoque lineal que implica una secuencia temporal de causa-efecto y que pierde de vista la complejidad del fenómeno, al prescindir del análisis de las fuerzas que provocan los factores que marcan la «atracción» y la «expulsión», muestra como un fenómeno aleatorio los «factores de atracción» o sólo se les atribuye la función de distribuidores entre los diferentes destinos que potencialmente existen (Roldán, 2011). Similares cortinas de humo lanzan quienes proponen que la pervivencia de los flujos migratorios se localiza en las redes transnacionales, las que confieren a los movimientos migratorios la posibilidad de que una vez iniciados se conviertan en procesos sociales autosostenidos, ya que reducen los costos económicos y psicológicos que acompañan a la migración. 
Estas perspectivas analíticas de la migración han creado gran confusión porque abordan aspectos que de modo incuestionable son rasgos presentes y necesarios para alcanzar su conocimiento, es decir, son verdades parciales que simplifican procesos. Es el caso de las condiciones de empleo y salario de los migrantes en sus países de origen y que son un elemento clave para el análisis teórico y en la definición de políticas públicas. El debate no es baladí si de lo que se trata es de identificar si dichas condiciones son las que propician las migraciones y su inserción en el mercado laboral del país de destino. Aceptar dicho planteamiento supondría que las relaciones capital/trabajo, en cuanto a las migraciones, no están comandadas por el primero y que el proceso de producción, comercialización y servicios ya no depende de las decisiones que asume el empresario, el capital, sino que las determina el asalariado, que además es inmigrante y en muchos casos carece de los papeles migratorios institucionalmente requeridos. Significa, también, que sólo existe una relación asimétrica entre países como México y Estados Unidos y no relaciones de poder y dependencia; que mujeres y hombres mexicanos son tan «ingeniosos» que en el transcurso de dos décadas más de 6 millones lograron burlar a la policía migratoria e introducirse al país más poderoso como indocumentados. Se diluye o desdibuja la responsabilidad y el compromiso que tienen los países receptores en el fenómeno migratorio laboral.

Cristina Blanco indica que la propuesta analítica del push e pull sugiere que si por un lado se localizan diversos factores negativos, vinculados a los países de origen (sobrepoblación, pobreza, escasez de tierra, salarios precarios, desempleo, violencia) y en los países de destino se ubica una realidad radicalmente distinta (riqueza, empleos mejor pagados, educación, salud, democracia), «la decisión de emigrar queda entonces limitada a las motivaciones individuales de los migrantes, presuponiendo una total libertad de acción» (Blanco, 2000:64). Tales planteamientos conminan a preguntarse por qué en un mundo donde la desigualdad se ha incrementado y más de 17 por ciento de la población mundial (mil 300 millones de personas) vive en situación de pobreza, sólo migra 3.3 por ciento (ONU, OPHI, 2019).

Que los migrantes toman decisiones, es otra verdad parcial. La pregunta es si son resoluciones tomadas con libertad o se hallan condicionadas por relaciones sociales, bases materiales, historia individual y colectiva, articulaciones entre individuos y su contexto. Me inclino por esto último, por identificar a los individuos que deciden migrar como personas que no son únicamente entes 
económicos y sencillos de conocer. Por el contrario, son mujeres y hombres que no sólo están compuestos por motivaciones económicas, que tienen consigo condiciones históricas y sociales y que no se encuentran al margen de la cultura; asimismo, no poseen toda la información económica, jurídica y social sobre las condiciones del tránsito y del destino. Recupero el pensamiento de Alexis de Tocqueville, Friedrich Nietzsche, Karl Marx, Thomas Carlyle y Søren Kierkegaard, quienes enunciaron algunas de las formas en las que la modernidad influye en las decisiones individuales del hombre, pero que no desconocen que el individuo moderno cuenta con la capacidad para discernir sus decisiones; de lograrlo, estará en condiciones de construir respuestas individuales y sociales que contemplen su contexto (Roldán, 2011).

Desde la heterodoxia, la perspectiva crítica del paradigma, se insiste en la urgente necesidad de contextualizar la «crisis mundial migratoria» y evidenciar su paralelismo e inserción en la profunda crisis sistémica de la globalización capitalista, reconocida como crisis civilizatoria por su carácter multidimensional (económica, política, social y ambiental), aunado a su manejo tendencioso para enfrentar coyunturas electorales en las que se advierte la necesidad de redefiniciones que buscan salidas a las contradicciones agudizadas por dicha globalización.

Al respecto, se propone ponderar que las conexiones de los mercados laborales entre los países de origen y los de destino están marcadas por el desarrollo desigual. Concerniente a la que existe entre Estados Unidos y los países latinoamericanos de donde proceden los principales flujos de este sistema migratorio, se caracteriza por la histórica dependencia y desigualdad estructural de estos últimos respecto de la hegemonía estadounidense, profundizada e institucionalizada con las reformas estructurales de varias generaciones, aplicadas durante los últimos cuarenta años. Muestra de ello es que el gobierno de México, en el primer semestre de 2019, aceptó darle continuidad a las — también históricasexigencias de que la frontera sur sea el gran muro contenedor de los flujos migratorios que transitan con dirección a ese país.

Dentro de ese modelo de desarrollo que surgió de la profunda crisis sistémica de los 1970, que acudía de nuevo a la globalización como un proceso que prometía la convergencia, el crecimiento de las exportaciones y la disminución de la migración, los países dependientes latinoamericanos intentaron participar en ese proceso, encabezado por los países altamente industrializados. Accedieron a él, pero en condiciones de "polizontes», de países de "segunda», que con el supuesto de lograr su inserción en la economía internacional en condiciones de igualdad, 
aceptaron el papel de maquiladores y proveedores de fuerza de trabajo barata. El costo fue la destrucción de la industrialización alcanzada en la etapa del modelo de sustitución de importaciones, el retroceso de los ya de por sí restringidos mercados laborales y el permanente deterioro salarial. Como nunca antes, se institucionalizó la libre movilidad de los capitales y de las mercancías y se colocó en absoluta incertidumbre la movilidad del trabajo, con graves consecuencias en los derechos humanos.

Resulta clara la incomprensión de los Estados involucrados, por razones ideológicas y políticas, de las causalidades de estos procesos, así como de sus profundas reticencias a construir políticas migratorias multilaterales que se acerquen a su gobernanza. Ello también es consecuencia de lo inoperativo de las estructuras de la supuesta "gobernanza global», pues no han contribuido en la construcción de experiencias modernas de la democracia a escala global (Mezzadra y Neilson, 2017). De ahí que se confirme que es ineludible analizar y explicar las causas y condiciones de las migraciones y la urgente necesidad de construir una respuesta social contundente que frene la xenofobia, el racismo y el clasismo en el que están inmersos sectores sociales cada vez más amplios.

\section{iA qué alude el concepto de la feminización de las migraciones?"1}

Los muros ideológicos abordados en el apartado previo y que constituyen profundos obstáculos para una comprensión ajustada a la realidad sobre las causas de las migraciones laborales, tienen una visión androcéntrica en la que los aspectos de género están ausentes o restringidos a aspectos anecdóticos. En contraste, las reflexiones propuestas con anterioridad, relativas al contexto y las causas de las migraciones laborales internacionales, conforman el tronco común, un marco general que comprende rasgos que atañen a mujeres y hombres y del cual se alimenta el análisis más fino al incluir la perspectiva de género. Su inclusión es primordial ya que hace referencia a una de las expresiones más importantes que de las migraciones internacionales y de autores como Stephen Castles y Mark J. Miller (2004), al hacer énfasis en las cinco tendencias generales de «la era de la migración» en su etapa contemporánea, la destacaron como la

${ }^{1}$ Es pertinente aclarar que esta investigación retoma el concepto del libro Las odiseas de Penélope. Feminización de las migraciones y derechos humanos, en específico, la «Introducción» elaborada en coautoría con María José Guerra, y el artículo "Mujeres en migración: principales controversias» (Roldán, Guerra y Pérez, 2017). 
feminización de la migración, que ha sido acompañada de una «mayor conciencia de la especificidad de las mujeres en las migraciones» (2004:22).

El alcance de su presencia en estos procesos ya es trascendente. De los 258 millones de migrantes internacionales (ONU, 2017) 54.2 por ciento son hombres y 45.8 por ciento mujeres. Un dato que desvela las particularidades de la migración de las mujeres es la tasa de participación laboral. Mientras que la de los hombres migrantes es de casi 75.5 por ciento y la de los hombres no migrantes o nativos de 75.2 por ciento (prácticamente igual), la de las mujeres migrantes es de 63.5 por ciento y la de las mujeres que no son migrantes es de 48.1 por ciento (ILO, 2018); es decir, en su mayoría las mujeres migrantes están incorporadas a los mercados laborales.

En adición, es relevante contextualizar el recuento anterior debido al discurso ampliamente difundido sobre la dimensión de la migración internacional, mismo que ha tenido un gran impacto social. En diversas investigaciones se ha corroborado que la percepción, en especial en los países de destino, no se corresponde con la realidad, de tal manera que tampoco es tarea menor identificar las reales dimensiones del fenómeno migratorio laboral y, sobre todo, el de las mujeres. En esa dirección y de acuerdo con estadísticas elaboradas por la Organización Internacional del Trabajo (OIT), el número de trabajadores a escala mundial fue de 3 mil 270.3 millones de personas (39 por ciento son mujeres y 61 por ciento hombres), de las cuales sólo 5 por ciento son trabajadores migrantes. Hay mil 271.3 millones de trabajadoras, pero únicamente 68 millones son mujeres migrantes, lo que significa que apenas representan 5 por ciento del empleo total de las mujeres y 2 por ciento del trabajo mundial total (OIT, 2019). Tal enumeración permite confirmar que la movilidad internacional no tiene el sentido pretendido y que sus alcances son regionales e identificables en los sistemas migratorios que histórica o coyunturalmente se configuran.

Ahora bien, las migraciones de las mujeres en el nivel mundial han estado y continúan con poca distancia de las que realizan los hombres. En ese sentido, deben atenderse los cuestionamientos de diversas autoras (Donato y Gabaccia, 2015; Paiewonsky, 2007; Oso, 2008) en cuanto a que si el término feminización hace relación a los aspectos cuantitativos (aparición o incremento absoluto). Las estadísticas corroboran que en las últimas siete décadas la variación en la presencia de mujeres en la migración apenas es de 1.8 décimas (ONU, 2018). Por tanto, es preciso distinguir la articulación que guardan las mujeres migrantes en esta etapa del capitalismo globalizante con condiciones sociales, oscilaciones 
demográficas, características de los procesos económicos productivos y reproductivos, y de imaginarios e ideologías que caracterizan los modelos de desarrollo.

En las últimas cuatro décadas, la corriente ampliamente mayoritaria de la migración femenina se asocia a tres grandes tendencias que observan los mercados laborales en los países de destino: generizados, terciarizados y flexibilizados. Dichas orientaciones concuerdan con los flujos migratorios de este periodo, lo que se refleja en su concentración en sectores económicos específicos y en las diferencias notables según el sexo. Asimismo, guardan correspondencia con diversas transformaciones sociodemográficas, de las que destaca la tendencia a la disminución de la población económicamente activa, por baja natalidad; el crecimiento de la participación de las mujeres nativas en la fuerza de trabajo; y el aumento del número de hogares biparentales, es decir, donde ambos miembros de la pareja son parte de la fuerza laboral. Así, las exigencias de fuerza de trabajo joven y de mujeres migrantes se incrementaron.

Otras condiciones que deben puntualizarse son las que hacen referencia a las particularidades observadas en las mujeres y en los países de donde provienen. La incorporación de las mujeres a la migración y al trabajo como inmigrantes no se contradice con su previa incorporación a los mercados laborales de sus países, lo que Sassen (2003) ha denominado la feminización de la proletarización. No se trata de una simple casualidad, se asocia directamente con los cambios y problemas para el acceso al empleo, así como el paulatino y persistente decremento de la capacidad adquisitiva de los salarios, que obligó a las familias a que aumentara el número de quienes aportan al ingreso familiar (jóvenes y mujeres). En ambos casos han sido, históricamente, parte del gran ejército industrial de reserva del capital: cuando se les requiere son incorporados y en su momento engrosan las filas del desempleo o son reenviadas al «cuidado del hogar» o a desarrollar su «capital humano». En este periodo la informalidad, la flexibilidad y la precarización que caracteriza a los mercados laborales requirieron de la inserción de aquellos que estuvieran dispuestos a «trabajar en sus horas libres» y que no contaran con experiencia en la defensa de sus derechos laborales.

Cabe resaltar que la consideración sobre el incremento de la incorporación de las mujeres a los mercados laborales no significa contradecir el planteamiento de Marcela Lagarde en cuanto a que «las mujeres siempre han trabajado». Por el contrario, la perspectiva feminista de género en las migraciones y de las cuales se destacan las aportaciones de Laura Pautassi, María José Magliano, Carmen 
Gregorio Gil, Mirjana Morokvasic, Patricia Pessar, Pierrette Hongdagneu-Sotelo, Natalia Ribas, Sara Mahler, Laura Oso, Saskia Sassen, Ruth Mestre, María José Guerra y Esther Torrado, parten del supuesto de que las relaciones socialmente construidas entre mujeres y hombres comprenden una premisa conceptual básica para el análisis de las relaciones de poder y desigualdad. De tal manera, es fundamental asumir que

la homologación de las actividades de la mujer con los hechos procreadores que le ocurren, como hechos naturales, conlleva a definir la esencia de cualquier trabajo femenino como sexual, biológica (no-humana). Por contagio se anula todo el trabajo femenino. Así, el trabajo de la mujer se constituye en mucho más que una característica sexual: es sexualidad femenina, queda subsumido y negado en la feminidadnaturaleza (Lagarde, 2015:113).

Las relaciones sociales de género en el sistema capitalista patriarcal actual permiten entender la etapa por la que transita la migración de las mujeres como un fenómeno social diferente, profundamente entretejido con la movilidad masculina, pero que destaca por las peculiaridades de su inserción en la reproducción social-productiva en los países de origen y destino. Lo anterior se refleja en las estadísticas proporcionadas por la Organización Internacional para las Migraciones (OIM, 2017): Norteamérica (51.5 por ciento mujeres y 48.5 por ciento hombres), Europa (52 y 48 por ciento), América Latina (50.4 y 49.6 por ciento) y Oceanía (51 y 49 por ciento). Como puede apreciarse, es ligeramente mayor el stock de mujeres respecto a los hombres. Son las diferencias existentes en Asia y África (15 y 6 por ciento más de hombres), las que explican por qué a escala mundial la distancia entre ambos es de 4.2 por ciento; continentes en los que las distancias de género en la participación económica y laboral son más profundas que en el resto.

En diversos trabajos he advertido que las migraciones de las mujeres al igual que las de los hombres, si bien en sus expresiones sociales han acompañado las diversas etapas de la historia de la humanidad, dicha movilidad se ha correspondido con realidades sociales e individuales, locales y generales, enlazadas con procesos y entornos que las condicionan (Roldán, 2011). Ello se refleja, entonces, en los contextos en los que se realizan desplazamientos humanos asociados a los orígenes de la humanidad y, posteriormente, en los que fueron apoyo-resultado de los primeros imperios. 
Concerniente a las migraciones en el capitalismo, se realizarán en situaciones diferentes. En opinión de Javier De Lucas, se requería de un Estado propio que asegurara la homogeneidad jurídico-política (el monopolio del derecho y la violencia legítima) y la cultural (lingüística, religiosa y étnica): «Eso es lo que muestran los trabajos de Burke, en línea con las tesis de Gellner, que es el nacimiento del Estado moderno lo que marca la ilusión de una cultura propia, pura, al poner fin al pluralismo cultural de la Baja Edad Media». Su aparición acompaña al surgimiento de la gran dicotomía ciudadano/extranjero y de la exclusión de los segundos en lo relativo a derechos (De Lucas, 1996:24).

A las condiciones generales de las migraciones internacionales contemporáneas, se deben incorporar aquellas que impone el capitalismo-patriarcal, que transforma a las mujeres en un gran ejército de reserva, en relación directa con el proceso de producción y reproducción sistémica nacional y mundial. La división del trabajo según el sexo no ha tenido una sola trayectoria en su movilidad entre el «hogar» y lo «público» y en su incorporación a los mercados laborales internos, su desplazamiento a través de las fronteras y su presencia en ellas, con movilidad o no (Roldán, 2017). El propósito no es hacer una revisión histórica de las diferentes etapas por las que ha transitado la incorporación de las mujeres en las migraciones internacionales en la modernidad; sólo se resaltará que las que acontecieron en la posguerra, para la reconstrucción de Europa y su reactivación económica, tuvieron como actores principales a los varones, con diversos momentos en los que las políticas migratorias contemplaron la reunificación familiar y la progresiva incorporación de las mujeres en varias actividades económicas (servicios personales, trabajo doméstico y comercio).

Finalmente, el concepto de feminización de las migraciones alude también a una nueva realidad individual y social de las mujeres que se representa en la autonomía social de la mujer frente al hombre y la familia en su decisión de migrar. Cambio vinculado con los rasgos que adquiere la relación capitalismo/patriarcado en esta etapa. La ideología de género no es estática y en su expresión contemporánea, asociada a los escenarios y símbolos de reclusión de las mujeres, contempla mutaciones que han tenido una manifestación esencial: la presencia de las mujeres en las industrias maquiladoras, turismo, comercio informal y formal y trabajo doméstico, en sus lugares de origen y como resultado de migraciones internas e internacionales. Tales modificaciones incluyen nuevas reglas patriarcales en las familias y comunidades, al igual que ideologías renovadas en el plano social sobre la movilidad fuera de los hogares, hacia otras ciudades y otros países. La 
información cuantitativa y cualitativa permite contrastar las migraciones de mujeres de las últimas cuatro décadas, como personas activas, que con su andar han comenzado o han consolidado autonomía frente a las anquilosadas disposiciones patriarcales que prevalecían en etapas anteriores y que circunscribían la movilidad de mujeres en los procesos de reunificación familiar o de acompañamiento, lo cual no implicaba que no se incorporaran al mercado laboral en el país de destino.

Lo anterior no sucede en condiciones fluidas, la contradicción permanente entre la exaltación de la movilidad y la consideración de que dicha movilidad entraña un drástico rompimiento con las viejas y rígidas estructuras patriarcales que recluyen a la mujer en «su hogar», se expresan de forma cotidiana y dan lugar a violaciones de derechos humanos con una clara perspectiva de género. Dichas violaciones no afectan por igual al conjunto de las mujeres, varía porque la experiencia migratoria es distinta según su participación en la estructura de clases, su pertenencia a alguna etnia, su edad, su preferencia sexual y su condición migratoria.

\section{Las mujeres latinas en el mercado laboral estadounidense}

La fuerte carga ideológica conservadora y xenófoba contenida en los discursos de los flujos migratorios en Estados Unidos provoca una imagen distorsionada en el imaginario social sobre las circunstancias en las que se desenvuelven las migraciones laborales. La política de cero tolerancia, el continuar con la construcción del muro, el anuncio de operativos de redadas del Servicio de Inmigración y Control de Aduanas de Estados Unidos (ICE, por sus siglas en inglés) para arrestar y deportar a los inmigrantes indocumentados, las presiones para que México contenga los flujos de centroamericanos, entre otras tantas atrocidades reactivadas por el gobierno estadounidense, se han convertido en herramientas con amplia efectividad, demostrada en las elecciones presidenciales de noviembre de 2016, con la finalidad de consolidar el voto duro conservador y de aquellos que han sido afectados por las políticas inherentes a la globalización. Redoblar los esfuerzos en aras de que en su campaña «Keep America Great» Donald Trump se mostrara como el más antimigrante, el más racista, el más misógino y el más proteccionista (America First), fue propiciado por su caída en la popularidad en varias encuestas en las que el límite máximo de sus calificaciones de aprobación 
se ha mantenido en 40 puntos hacia abajo, en específico en estados como Michigan, Wisconsin y Pensilvania, cuya relevancia fue fundamental para su triunfo en el anterior proceso electoral.

En ese contexto tan adverso, żcuál es el nivel de presencia de mujeres y hombres latinos en Estados Unidos? La información presentada se sustenta en la base de datos IPUMS-USA, que se construyó a partir de la American Community Survey ${ }^{2}$ acerca de la movilidad femenina latinoamericana. Desde la década de 1970 la Oficina de Censos de Estados Unidos utilizó el término «latino» para contabilizar a las personas que viven en este país, pero que nacieron en América Latina, ${ }^{3}$ o que al menos uno de sus padres nació en esa región. Es ampliamente reconocido entre los estudios del tema migratorio y por organismos internacionales (OIT y la Organización de las Naciones Unidas) que las deficiencias en las estadísticas migratorias siguen siendo importantes. En consecuencia es necesario advertir que los datos comprenden una fotografía del fenómeno proporcionada por los censos, que revela el volumen acumulado (stock); no obstante, no dan cuenta del flujo y de la velocidad con la que tales procesos están cambiando. Asimismo, denotan sólo un acercamiento a la realidad, puesto que es posible inferir que hay una subestimación en los datos, en virtud de la magnitud de los inmigrantes indocumentados que no estuvieron dispuestos a ser censados.

A fin de dar respuesta a la interrogante, no se realizará una evaluación de la economía de Estados Unidos, ni de sus perspectivas para los próximos meses (incluso el Fondo Monetario Internacional ha bajado las expectativas de crecimiento de la economía mundial en 2019 y ha previsto la desaceleración de las economías industrializadas). Por ende, únicamente se destacarán las condiciones del empleo, que están cercanas a su mejor mínimo en 18 años (casi al nivel del año 2000), situación que mantiene una relación directa con sus requerimientos de fuerza de trabajo inmigrante. Referente a la migración de latinos se valida la afirmación de

${ }^{2}$ IPUMS-USA es un proyecto de la Universidad de Minessota que concentra y homologa los microdatos que se generan en los censos decenales desde 1850 y de la encuesta American Community Survey desde el año 2000.

${ }^{3}$ El término latino contempla a personas provenientes de las cuatro subregiones latinoamericanas: América del Norte (México, América Central, Belice, Guatemala, El Salvador, Honduras, Nicaragua, Costa Rica y Panamá), América del Sur (Argentina, Bolivia, Brasil, Chile, Colombia, Ecuador, Guayana, Paraguay, Perú, Surinam, Uruguay y Venezuela) y el Caribe (Cuba, República Dominicana, Haití, Barbados, Trinidad y Tobago y Jamaica). Cabe precisar que en esta investigación sólo tomará en cuenta a aquellas mujeres que su lugar de nacimiento sea un país de la demarcación y que vivan en Estados Unidos. 
Raúl Delgado Wise y Selene Gaspar Olvera: «El movimiento de la migración mexicana a Estados Unidos se correlaciona positivamente con la tasa de desempleo en ese país; es decir, uno de los motores principales de la emigración mexicana ha sido la demanda de empleo» (2017:11).

El comportamiento del desempleo de los nativos mantiene la misma tendencia que la de los inmigrantes latinos. En 24 años, el nivel de desempleo en el total de inmigrantes y en el de las mujeres siempre es mayor que el de la población nativa (gráficas 1 y 2). Pese a la sombría situación de xenofobia en ese país, la presencia de las mujeres y hombres latinos inmigrantes no ha disminuido; ha mantenido una tasa de crecimiento, si bien no al ritmo de la observada en los 1990 y a principios de siglo, a causa de las crisis en 2001 y 2008 de la economía estadounidense. Las cifras correspondientes a las mujeres se han igualado a las de los hombres latinos (véase gráfica 3). La disminución de la tasa de crecimiento de la migración de latinos hacia Estados Unidos, a partir de la primera década del siglo XXI, corrobora la hipótesis de que son las condiciones de su proceso de acumulación las que los obligan a movilizarse. La crisis no fue exclusiva de la economía estadounidense, en 2009 en México el decrecimiento de la economía fue de 6.5 por ciento, con fuertes impactos en el empleo formal y en los salarios; sin embargo, el empeoramiento no se tradujo en una mayor incorporación de mexicanos en el flujo migratorio, sino que disminuyó por la caída en el empleo del vecino país.

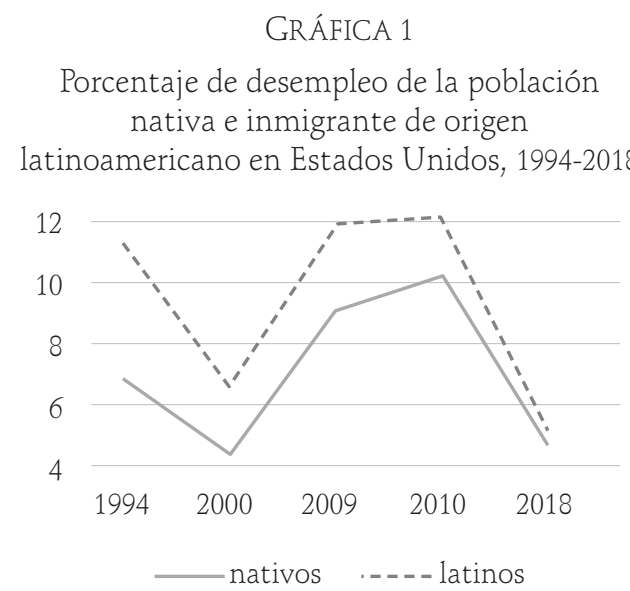

Fuente: elaborado por Daniela Castro Alquicira con datos de IPUMS CPS, 2018. 


\section{GRÁFICA 2}

Porcentaje de desempleo de mujeres nativas e inmigrantes de origen latinoamericano en Estados Unidos, 1994-2018

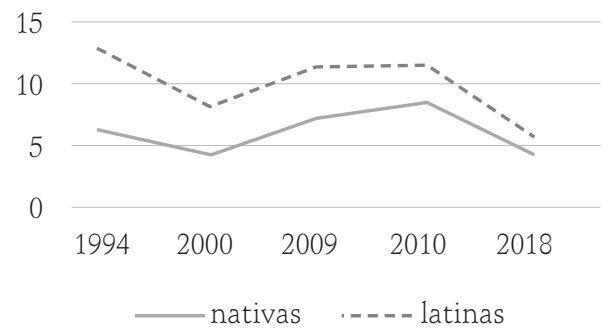

Fuente: elaborado por Daniela Castro Alquicira con datos de IPUMS CPS, 2018.

\section{GRÁFICA 3}

Inmigrantes latinoamericanos en Estados Unidos según sexo, 1994-2018

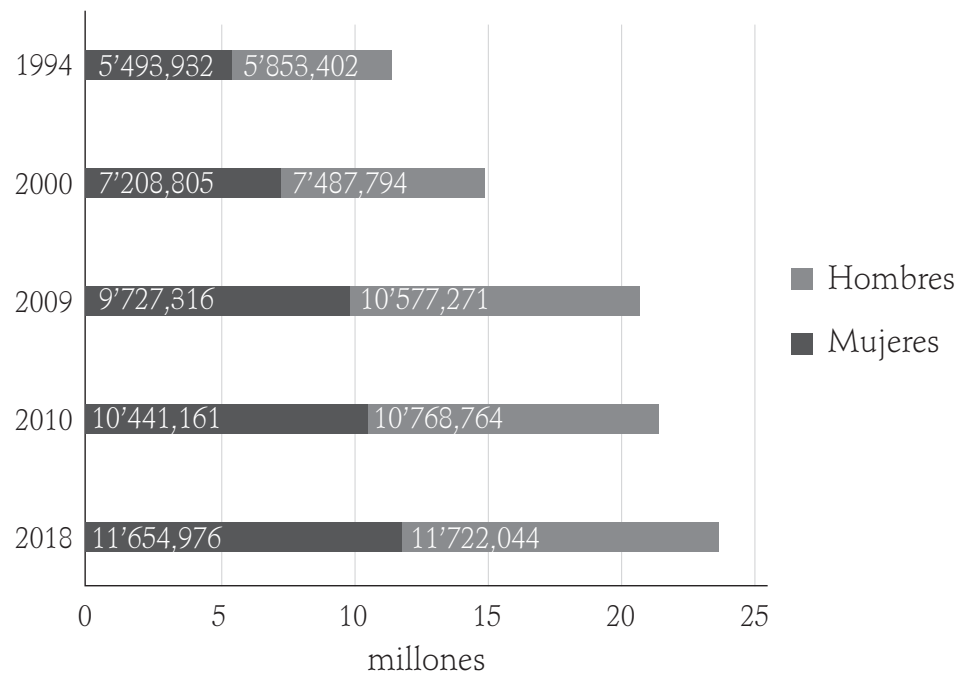

Fuente: elaborado por Daniela Castro Alquicira, con datos de IPUMS CPS, 2018.

El aumento de mujeres latinas en Estados Unidos se vincula con el incremento de su presencia en los mercados laborales. En la gráfica 4 se aprecia que en 24 años la proporción de latinas se ha duplicado, gracias a la profunda reconversión de su economía y del mundo laboral. Las mujeres conforman la reserva de mano de obra ideal para las nuevas ocupaciones, donde se les confina a escalas 
de salarios menores (Berman, 1998) y en empleos cada vez más precarios e informales. Se confirma que las mujeres se han convertido en actores clave en dichos mercados (Roldán y Castro, 2018).

\section{GRÁFICA 4}

Proporción de latinas en la fuerza laboral femenina, Estados Unidos, 1994-2018

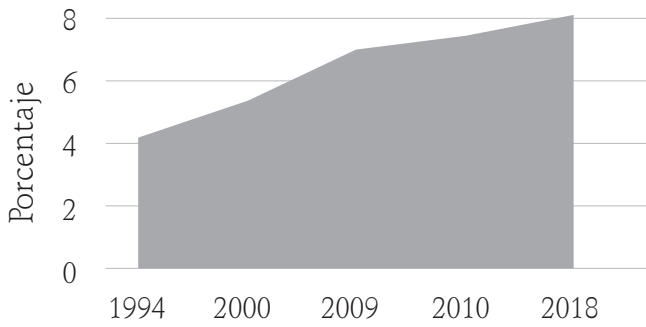

Fuente: elaborado por Daniela Castro Alquicira, con datos de IPUMS CPS, 2018.

La extranjerización de los mercados laborales es un fenómeno que ha evidenciado todavía más la importancia de los trabajadores inmigrantes en ciertos sectores económicos. En escala global se encuentran concentrados en más de 70 por ciento en el sector servicios, de los cuales los trabajadores domésticos alcanzan 8 por ciento; alrededor de 20 por ciento labora en la industria, la construcción y la agricultura. En lo relativo al trabajo doméstico, 67 millones de personas lo realizan en todo el mundo; de ellas, 17 por ciento es inmigrante (11.5 millones) y de éstos 8.5 millones son mujeres (74 por ciento) (OIT, 2017). Cerca de 13 por ciento es trabajadora doméstica y 87 por ciento de las mujeres trabajadoras migrantes internacionales labora en otras actividades. En los sectores en los que participan las mujeres latinas en Estados Unidos, 82 por ciento se ubica en el sector servicios y 11 por ciento en trabajo doméstico (véase cuadro 1).

Son diez las principales actividades de ocupación en las que se insertan las latinas en Estados Unidos y que sobresalen en los censos: trabajadoras domésticas (10.7 por ciento), auxiliares y asistentes de enfermería (7.4 por ciento), cocineras ( 4.5 por ciento), intendentes ( 4 por ciento), niñeras ( 3.4 por ciento), cajeras ( 3 por ciento), jornaleras y empacadoras (2.4 por ciento), secretarias ( 2 por ciento), meseras (2 por ciento), empleadas de venta al por menor (1.8 por ciento). 
CUADRO 1

Sectores de actividad de las mujeres en Estados Unidos, 2018

\begin{tabular}{lrr} 
Sectores & Nativas & Latinas \\
\hline Agricultura, silvicultura y pesca & 1.52 & 2.56 \\
Minería & 0.15 & 0.00 \\
Construcción & 1.51 & 1.57 \\
Manufactura & 5.76 & 10.17 \\
Comunicaciones y transportes & 3.78 & 3.50 \\
Ventas al mayoreo & 1.46 & 2.51 \\
Ventas al menudeo & 19.02 & 19.21 \\
Finanzas, seguros y bienes raíces & 7.81 & 4.16 \\
Servicios financieros y administrativos & 5.42 & 11.64 \\
Servicios personales & 3.84 & 10.09 \\
Recreación y entretenimiento & 2.20 & 1.27 \\
Servicios profesionales y relacionados & 43.09 & 31.10 \\
Administración pública & 4.42 & 2.23 \\
\hline Total & 100.00 & 100.00
\end{tabular}

Fuente: elaborado por Daniela Castro Alquicira con datos de IPUMS CPS, 2018.

Las estadísticas institucionales alertan sobre la necesidad de profundizar en el conocimiento más específico de las condiciones del trabajo en ese amplio sector servicios y que no son iguales a las que se llevan a cabo en los servicios domésticos, de la reproducción social, servicios de proximidad, servicios a la vida diaria y las cadenas de cuidados, en los que se ha puesto mayor atención en investigaciones relevantes referidas no sólo a las latinas en Estados Unidos, sino las que se producen en Europa y en la movilidad sur-sur.

\section{Conclusiones}

Al abordar la feminización de las migraciones se cuestiona la percepción social androcéntrica y sexista que invisibiliza la presencia de las mujeres o la representa subordinada o adherida a la protagonizada por los hombres. Es entendida como un proceso clave en las singularidades que exhiben las dinámicas migratorias, a partir de la reestructuración capitalista que tuvo su auge en los últimos 40 años. La hipótesis que orienta la investigación es que el detonante de dicha 
feminización son las grandes tendencias de los mercados laborales de los países de destino, lo cual no significa ignorar o asignarle un papel secundario al contexto y a las características estructurales, sociales e individuales de los países de origen.

Las relaciones sociales de género en el sistema capitalista patriarcal actual hacen factible entender la etapa por la que transita la migración de las mujeres como un fenómeno social diferente, profundamente entretejido con la movilidad masculina. De similar importancia es la reflexión que destaca sus expresiones concretas en cuanto a los rasgos y peculiaridades de su inserción en la reproducción social-productiva en los países de origen y destino.

Debe distinguirse la articulación que guardan en esta etapa del capitalismo globalizante las condiciones sociales, las oscilaciones demográficas, las características de los procesos económicos productivos y reproductivos y de los imaginarios e ideologías de los modelos de desarrollo que vinculan dichos contextos. El concepto de feminización de las migraciones alude también a una nueva realidad individual y social de las mujeres, que se materializa en la autonomía social de la mujer frente al hombre y la familia en su decisión de migrar.

Así, el artículo recupera aspectos esenciales del debate con el mainstream, con la ortodoxia dominante en las teorías migratorias, sobre las causalidades de las migraciones laborales internacionales, en el entendido de que son consideradas el tronco común, un marco general, que al incluir la perspectiva de género nutre el análisis. La información desglosada corrobora que la corriente ampliamente mayoritaria de las mujeres en migración laboral se vincula a las tendencias globales de los últimos 40 años. Su incorporación ocurre en empleos terciarizados, flexibles y generizados. Además, se confirma que la inserción de las mujeres latinas a los mercados laborales estadounidenses se corresponde con las necesidades de los sectores económicos a los que se han dirigido. El capitalismo globalizante no es, de ningún modo, armonioso. En ese sentido, el discurso xenófobo, por claras orientaciones políticas, es contradictorio con las necesidades del capital, pero es este último el que dicta la última palabra.

Es pertinente aclarar que en el trabajo quedó ausente el análisis de un asunto que ha sido objeto de gran atención en las investigaciones sobre la feminización de las migraciones y que ahora sólo fue enunciado: el nivel de contribución de la migración de las mujeres trabajadoras en su rompimiento con las estructuras patriarcales, tema central en el estudio desde una perspectiva de género. Se carece de los elementos para realizar afirmaciones concluyentes, pero sí se cuenta con 
algunos que permiten avanzar en una dirección que observe las principales tendencias. Es vital distinguir los planos de análisis que pueden orientar acerca de la magnitud y la trascendencia del cambio social logrado a partir de la incorporación de las mujeres a la migración laboral.

Finalmente, la migración por sí misma no otorga a los sujetos (mujeres y hombres) una comprensión crítica de la realidad ni elimina aquellos prejuicios en los que está sumergida la ideología capitalista-patriarcal que distorsionan, tergiversan y ocultan la realidad de las mujeres migrantes.

\section{Agradecimientos}

La autora agradece el apoyo en la sistematización de la información estadística a Daniela Castro Alquicira, técnica académica del Instituto de Investigaciones Económicas, UNAM.

\section{Referencias}

Blanco, Cristina (2000), Las migraciones contemporáneas, Madrid, Editorial Alianza.

Braverman, Harry (1998), Trabajo y capital monopolista: la degradación del trabajo en el siglo XX, México, Nuestro Tiempo.

Castles, Stephen y Mark J. Miller (2004), La era de la migración. Movimientos internacionales de población en el mundo moderno, México, Miguel Ángel Porrúa/Cámara de Diputados LIX Legislatura.

De Lucas, Javier (1996), Puertas que se cierran: Europa como fortaleza, Barcelona, Icaria.

Delgado Wise, Raúl y Selene Gaspar (2017), «Pacto mundial: migrantes mexicanos frente al espejo de la economía estadounidense», Migración y Desarrollo, 15(29).

Donato, Katherine y Gabaccia, Donna (2015), Gender and international migration. From the Slavery Era to the Global Age, Nueva York, Russel Sage Foundation.

Huntington, Samuel (2004), Who are we? The challenge to America's national identity, Nueva York, Simon and Schuster.

International Labour Organization (ILO) (2018), ILO Global Estimates on International Migrant Workers. Results and Methodology, Suiza, ILO.

Legarde y de los Ríos, Marcela (2015), Los cautiverios de las mujeres. Madresposas, monjas, putas, presas y locas, México, Siglo XXI Editores. 
Mezzandra, Sandro y Brett Neilson (2017), La frontera como método, Madrid, Traficantes de sueños.

Organización de las Naciones Unidas (ONU) (2017), Tendencias en el stock de migrantes internacionales, 2015, Nueva York, Departamento de Asuntos Económicos y Sociales, en: http://www.un.org/en/development/desa/population/index.shtml

Organización de las Naciones Unidas y OPHI (2019), Global multidimensional poverty index 2019. Illuminating inequalities, Nueva York, ONU-OPHI.

Organización Internacional del Trabajo (OIT) (2017), Informe IV. Migración laboral: nuevo contexto y desafíos de gobernanza, Conferencia Internacional del Trabajo, 106a reunión, OIT, Suiza. (2019), Perspectivas sociales y del empleo en el mundo, Suiza, OIT.

Oso, Laura (2008), «Migración, género y hogares transnacionales», en Joaquín García y Joan Lacomba (coords.), La Inmigración en la sociedad española. Una radiografía multidisciplinar, España, Ediciones Ballatierra.

Paiewonsky, Denise (2007), Feminización de la migración, Documento de Trabajo 1, Serie Género Migración y Desarrollo, Santo Domingo, INSTRAW/ONU.

Roldán, Genoveva (2011), "Las migraciones laborales internacionales y algunos de sus mitos», en Ana M. Aragonés (coord.), Mercados de trabajo y migración internacional, México, Instituto de Investigaciones Económicas IIEc-UNAM.

Roldán, Genoveva, María José Guerra y Nancy Pérez (2017), Las odiseas de Penélope. Feminización de las migraciones y derechos humanos, España-México, IIEc-UNAM/Universidad de la Laguna/Instituto Canario de Igualdad/Ministerio de economía, industria y competitividad del gobierno de España.

Roldán, Genoveva y Daniela Castro Alquicira (2018), "Latinas en el mercado laboral estadounidense del cuidado, 1990-2014», en María Luisa González y Patricia Rodríguez (coords.), Migración y precariedad femenina en América Latina. Propuesta de política económica, México, IIEc-UNAM.

Ruggles, Steven, Sarah Flood, Ronald Goeken, Josiah Grover, Erin Meyer, Jose Pacas y Matthew Sobek (2018), IPUMS USA: Version 9.0 [dataset], Minneapolis, Minnesota, Integrated Public Microdata Series USA (IPUMS USA), en https://doi.org/10.18128/D010.V9.0 Sassen, Saskia (2003), Contra geografías de la globalización. Género y ciudadanía en los circuitos transfronterizos, Madrid, Traficantes de Sueños.

United Nations-Department of Economic and Social Affairs (UN DESA) (2017), International migration report 2017, Nueva York, UN DESA, Population Division. 